\title{
Immunoglobulin G4-related sclerosing cholangitis
}

\author{
George Goodchild, Stephen P. Pereira, and George Webster
}

Department of Gastroenterology, University College Hospital, London, UK

Received: January 9, 2018

Accepted: February 6, 2018

\section{Correspondence to}

George Webster, M.D.

Department of Gastroenterology, University College Hospital, 250

Euston Rd, London NW1 2PG,

UK

Tel: $+44-2034567890$

Fax: $+44-2034479218$

E-mail: George.websterı@nhs.net
Immunoglobulin $\mathrm{G}_{4}$-related disease ( $\mathrm{IgG}_{4}-\mathrm{RD}$ ) is a systemic fibroinflammatory condition of which IgG4-related sclerosing cholangitis (IgG4-SC) is the biliary manifestation. In this review, we provide an overview of IgG4-RD, with a focus on the biliary manifestations. In particular, we describe the important differential diagnoses of $\mathrm{IgG}_{4}-\mathrm{SC}$, namely, primary sclerosing cholangitis and cholangiocarcinoma, outline diagnostic criteria for $\mathrm{IgG}_{4}-\mathrm{SC}$, provide insight into possible pathophysiological mechanisms underlying the disease and discuss short and long-term management options of this recently described disease.

Keywords: Immunoglobulin $\mathrm{G}_{4}$ related disease; Immunoglobulin $\mathrm{G}_{4}$ cholangiopathy; Autoimmune pancreatitis; Cholangitis, sclerosing; Biliary stricture

\section{INTRODUCTION}

Immunoglobulin $\mathrm{G}_{4}$-related disease (IgG4-RD) is a multi-system immune system-mediated disorder of unknown etiology. IgG4 sclerosing cholangitis (IgG4$\mathrm{SC}$ ) is the biliary manifestation of IgG4-RD. Awareness of IgG4-SC is important as it may mimic other benign and malignant conditions, including primary sclerosing cholangitis (PSC) and cholangiocarcinoma (CCA), but the management of the various conditions differs greatly. The diagnostic criteria for IgG4-SC include clinical, radiological, serological, and histological features. Corticosteroids (with or without immunosuppressants) are the mainstay of $\mathrm{IgG}_{4}$-SC treatment, although the optimal treatment regimen for maintenance of disease remission remains unclear. Rituximab, which depletes CD2o-positive plasmablasts, is a novel treatment option that may induce and maintain remission in patients with $\mathrm{IgG}_{4}$-SC who have failed treatments with conventional agents. This review offers a broad overview of IgG4-RD and discusses the specific diagnostic and therapeutic challenges.

\section{IgG4-RELATED DISEASE}

IgG4-RD is a recently defined multisystem fibroinflammatory condition that has been described in almost every organ [1]. Many diseases that have long been viewed as specific entities (e.g., Kuttner's tumor, Mikulicz's disease, retroperitoneal fibrosis, and Riedel's thyroiditis) are now considered to be part of the IgG4-RD spectrum. Classic sites of involvement include the pancreas, biliary tract, salivary and lacrimal glands, kidneys, thyroid gland, lungs, retroperitoneum, and aorta [2]. The clinical features depend largely on the organ(s) involved, although the most common presentation is the development of a tumefactive (mass) lesion or swelling of one or more organs, with misdiagnosis of malignancy being commonplace [3]. In one early series, $18 / 53$ patients (34\%) undergoing surgical resection of presumed pancreatobiliary malignancies were found to have IgG4-RD [4].

\section{Pathogenesis}

The pathophysiological mechanisms underlying IgG4RD remain incompletely understood. Current evidence suggests roles for both autoimmunity and allergy (in genetically susceptible individuals) in disease develop- 
ment. Several human leukocyte antigen (HLA) association studies in Japan, the United Kingdom (UK), and Korea have identified region-specific HLA molecules and other immunoregulatory genes as determinants of disease susceptibility [5]. It is likely that abnormal innate and acquired immunity, reduced levels of regulatory T-cells, and specific B-cell responses, are all involved in disease development [6]. Indeed, B-cell depletion following rituximab-induced disease remission was the first pathophysiological finding of $\mathrm{IgG}_{4}-\mathrm{RD}[7]$. Affected organs are heavily infiltrated with both $\mathrm{T}$ and $\mathrm{B}$ lymphocytes, indicating that an antigen-mediated response may be in play. The fact that oligoclonal $\mathrm{IgG}_{4}$ is present in the cerebrospinal fluid of patients with IgG4-related pachymeningitis further supports the existence of an antigen-driven immune response in such patients [8]. Later studies used next-generation sequencing to identify oligoclonal expansions of somatically hypermutated $\mathrm{IgG}_{4}+\mathrm{B}$-cell clones from affected tissue and blood [9]. An IgG4 antibody is presumed to be involved in the development of IgG4-RD, due to the characteristic infiltrates found in involved tissue. However, it remains to be determined whether the antibody is directly pathogenic, plays a protective role, or is merely an incidental marker of an aberrant inflammatory response [10]. Other cell lines of interest in terms of IgG4-RD pathophysiology include $\mathrm{CD}_{4}+$ cytotoxic $\mathrm{T}$ lymphocytes, which are expanded in both tissues and the plasma of patients with $\mathrm{IgG}_{4}-\mathrm{RD}$ and which decline in number following rituximab treatment [11], and $\mathrm{T}$ follicular helper cells (Tfh cells). In healthy individuals, such cells control
B-cell differentiation but, in IgG4-RD patients, the cells contribute to class-switching and expansion of $\mathrm{IgG}_{4+}$ plasma cells [12-14].

\section{Diagnosis}

IgG4-RD appears to be a disease of predominantly older males (the male:female ratio, 3:4) and the mean age of onset is 70 to 80 years [15]. These demographics seem to be particularly true of a form of autoimmune pancreatitis (AIP): type $1 / I_{\text {IgG }}$-related pancreatitis (IgG4-RP). In 2011, the prevalence of AIP in Japan was 4.6/100,000 of the population [16]. The diagnosis of $\mathrm{IgG}_{4}-\mathrm{RD}$ remains problematic because of the diverse range of clinical presentations, poor recognition by physicians, and misdiagnosis. It is thus challenging to give accurate estimations of the disease prevalences in other organs [15].

Several diagnostic criteria have been developed to aid in the diagnosis of IgG4-RD. The most widely used are the HISORt (Histology, Imaging, Serology, other ORgan involvement and response to therapy) criteria of the Mayo Clinic. This tool was initially used to diagnose AIP but can be adapted to diagnose IgG4-SC (Table 1) [4]. The required features include positive imaging findings, characteristic histological presentations, elevated serum $\mathrm{IgG}_{4}$ levels, extra-biliary organ involvement, and a response to steroid treatment [17]. Serum $\mathrm{IgG}_{4}$ levels are elevated $(>1.4 \mathrm{~g} / \mathrm{L})$ in up to $70 \%$ of all patients [18] and may correlate positively with the number of organs involved [19]. It is well-documented that elevated serum IgG4 levels are of limited utility when employed to distinguish IgG4-RD from inflammatory and malignant

Table 1. HISORt criteria for the diagnosis of $\mathrm{IgG}_{4}-\mathrm{SC}$

\begin{tabular}{|c|c|}
\hline Histology bile ducts & $\begin{array}{l}\text { Lymphoplasmacytic sclerosing cholangitis on resection: LP infiltrate > } 10 \text { IgG4+ cells } / \mathrm{hpf} \text {, } \\
\text { storiform fibrosis, phlebitis }\end{array}$ \\
\hline Imaging bile ducts & $\begin{array}{l}\text { One or more strictures involving intra- or extra-hepatic bile ducts } \\
\text { Fleeting/migrating biliary strictures }\end{array}$ \\
\hline Serology & $\mathrm{IgG}_{4}>2 \times \mathrm{ULN}$ \\
\hline Other sites & $\begin{array}{l}\text { Pancreas: classic features of AIP on imaging or histology } \\
\text { Retroperitoneal fibrosis } \\
\text { Renal: parenchymal low-attenuation lesions } \\
\text { Salivary/lacrimal gland enlargement }\end{array}$ \\
\hline Rt response to treatment & Normalisation of LFTs or resolution of biliary stricture \\
\hline
\end{tabular}

Adapted from Ghazale et al., with permission from Elsevier [4].

IgG4-SC, immunoglobulin G4 sclerosing cholangitis; LP, lymphoplasmacytic; hpf, high powered field; ULN, upper limit of normal; AIP, autoimmune pancreatitis; Rt, response to treatment; LFT, liver function test. 
disease [19-21]. A large UK cohort study recently found that only $22.4 \%$ of patients with elevated serum $\mathrm{IgG}_{4}$ levels (>1.4 g/L; $\mathrm{n}=264)$ met the IgG4-RD diagnostic criteria [22]. Doubling the cutoff to $>2.8 \mathrm{~g} / \mathrm{L}$ increased specificity from $85 \%$ to $96 \%$ but with an associated reduction in sensitivity (to $57 \%$ from $83 \%$ ). The positive predictive value of $45 \%$ was not impressive. Furthermore, serum $\mathrm{IgG}_{4}$ levels are elevated in up to $5 \%$ of healthy controls [19-21].

Histopathology is the gold standard for diagnosis of IgG4-RD, often supported by elevations in the numbers of blood and/or tissue IgG4-positive plasma cells. The three histological hallmarks of IgG4-RD are: IgG4-positive lymphoplasmacytic tissue infiltrates; storiform fibrosis (exhibiting a cartwheel or whorled appearance); and obliterative phlebitis (partial or complete obliteration of a vein) [1]. The presence of at least two of these histological features supports a diagnosis of IgG4-RD, with the cutoffs for the number of IgG4-positive plasma cells being defined for each organ. The IgG4:IgG ratio must be $>40 \%[1]$. It should be noted that lymphoplasmacytic infiltrates coupled with high numbers of IgG4-positive plasma cells are evident in a those with a variety of inflammatory and malignant conditions $[23,24]$. Therefore, the histology should be cautiously interpreted in the context of the clinical picture as well as the serological and radiological investigations [15].

Computed tomography (CT) and magnetic resonance imaging (MRI) both detect organ enlargement and therefore play important roles in defining the extent of disease and assessing the response to treatment. Positron emission tomography (PET)/CT detects clinically silent organ involvement; a recent study found that $90 \%$ of patients with "single-organ involvement" initially enrolled in an IgG4-RD had multi-organ disease on PET/ CT using the glucose analog 2-[(18)F]-fluoro-2-deoxy-dglucose (FDG), highlighting the value of PET in detecting other organ involvement [25].

\section{Treatment}

The aims of IgG4-RD treatment are to improve symptoms, restore organ function, and prevent progression to irreversible fibrosis. Treatment is usually required by patients in whom IgG4-RD is accompanied by organ involvement, but, in patients with mild disease (for example, asymptomatic lymphadenopathy), watchful wait- ing may be reasonable. Corticosteroids are the mainstay of treatment; the first prospective, randomized, placebo-controlled trial of such drugs in patients with AIP type 1 disease has recently been published. In this study, remission was induced in both groups and corticosteroids were then withdrawn (at 26 weeks) in 19/49 patients but maintenance treatment continued for 36 months in 30/49. The relapse rate was significantly lower in the maintenance group, $7 / 30$ patients $(23.3 \%)$ relapsed compared with $11 / 19(57.9 \%)$ in the cessation group $(p=0.011)$ [26]. A typical adult induction regimen featured prednisolone 30 to $40 \mathrm{mg} /$ day for 4 weeks, followed by tapering every 2 weeks (depending on the response) by $5 \mathrm{mg} /$ day over 3 to 4 months. A response was usually evident within 4 to 6 weeks as evidenced by improved organ function, radiological improvement, a decrease in serum the $\mathrm{IgG}_{4}$ level, and improvement of symptoms. It is unclear whether maintenance therapy should be commenced in all patients or solely in those with multiorgan involvement and/or relapse. Maintenance options include single-agent low-dose corticosteroids (ideally at no more than $5 \mathrm{mg}$ /day), with or without second-line immunosuppressive therapy [27] featuring either azathioprine 1.5 to $2 \mathrm{mg} / \mathrm{kg} /$ day or mycophenolate mofetil [28]. A novel (promising) option is rituximab, an agent that depletes CD2o-positive plasmablasts. This drug has been given to patients who were steroid-intolerant or dependent, and to those who failed to enter remission when on steroids only. The drug effectively induced and maintained remission in IgG4-RD patients, but was associated with high rates of serious infections [29-31].

A recent systematic review and meta-analysis (SRMA) of 62 studies involving 3,034 patients emphasized the key role for corticosteroid treatment; 1,186 of 1,220 patients (97\%) who received steroid monotherapies experienced therapeutic responses, but these were incomplete in $35 \%$ of patients. The study also evaluated relapse treatments. Relapses were reported in 464/1,395 patients(33\%), despite the typically short follow-up periods. Corticosteroids were effective in 219/231 (95\%) of such patients, in $56 / 69$ (81\%) of those treated with azathioprine, in 16/22 (72\%) of those treated with other immunosuppressive agents, and in all nine cases treated with rituximab (100\%) [32]. In patients with AIP type $1 / \operatorname{IgG}_{4}-\mathrm{RP}$, any associated proximal IgG4-SC predicted an increased risk of post-steroid relapse [33]. 


\section{Prognosis}

The natural history of IgG4-RD remains unclear; longterm data are lacking. Some patients improve spontaneously; many exhibit a benign disease course; but others relapse several times. There is some evidence that prompt treatment may be associated with better outcomes, possibly due to fewer inflammatory and fibrotic complications [5]. The risk of any malignancy (particularly lymphomas) appears to be increased by $>2$-fold in patients with $\mathrm{IgG}_{4}-\mathrm{RD}$ compared with age- and sexmatched controls [34].

\section{IgG4-RELATED SCLEROSING CHOLANGITIS}

The biliary manifestation of IgG4-RD is IgG4-SC. This the most common extra-pancreatic manifestation of AIP type 1 disease; estimates of the prevalence vary widely. In UK and United States cohorts, the prevalence of isolated $\mathrm{IgG}_{4}-\mathrm{SC}$ was $<10 \%$ that of IgG4-RD [4,18], and $60 \%$ to $80 \%$ of AIP patients had evidence of IgG4-SC [35,36]. IgG4-SC has a preponderance for older males and may exhibit a variety of presentations; a very recent retrospective study of 527 patients with $\mathrm{IgG}_{4}$-SC in Japan found that $35 \%$ presented with jaundice and $13 \%$ pruritus, whereas $28 \%$ were asymptomatic [37]. Other presentations include abdominal pain and weight loss.

\section{Diagnosis}

The HISTORt criteria can be used to diagnose IgG4-SC. Serum $\mathrm{IgG}_{4}$ levels are elevated in about $75 \%$ of patients [38]. Initial imaging may include abdominal ultrasonography, which may reveal biliary dilatation, stones, or a pancreatic mass lesion suggesting either AIP or a pancreatic tumor. Cross-sectional imaging is mandatory when further evaluating biliary strictures, bile duct wall thickening, and inflammation (enhancement); the involvement of other organs, and lymphadenopathy [39]. Magnetic resonance cholangiopancreatography (MRCP) and endoscopic retrograde cholangiography (ERC) are both important when evaluating IgG4-SC. MRCP may reveal segmental strictures, lower bile duct strictures, and long strictures with pre-stenotic dilatations; all are more common in $\mathrm{IgG}_{4}-\mathrm{SC}$ than CCA patients. Any part of the biliary tree can be affected in IgG4-SC patients; however, the lower bile duct tends to be the most com-

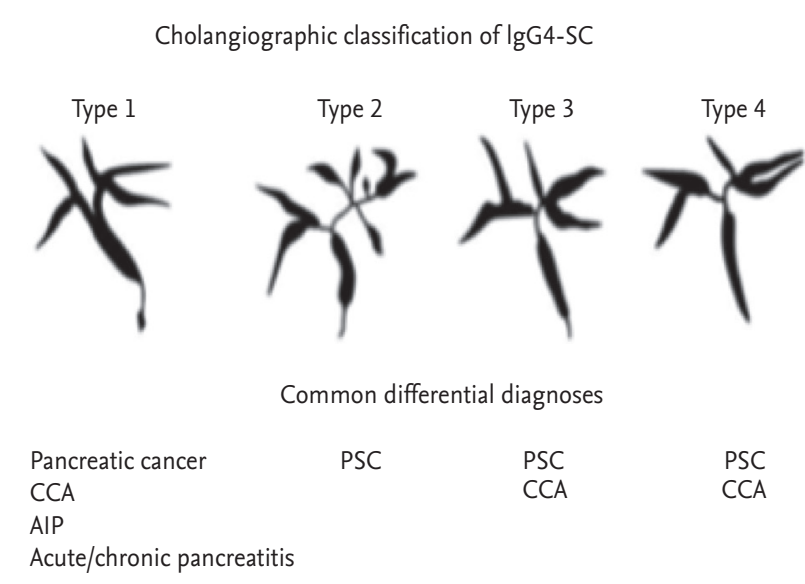

Figure 1. Cholangiographic classification of immunoglobulin $\mathrm{G}_{4}$ sclerosing cholangitis (IgG4-SC). Adapted from Nakazawa et al., with permission from Wolters Kluwer Health, Inc. [40]. PSC, primary sclerosing cholangitis; CCA, cholangiocarcinoma; AIP, autoimmune pancreatitis.

monly involved (often in conjunction with AIP type 1/ IgG4-RP). IgG4-SC has been classified into four subtypes based on the anatomical positions of the strictures (Fig. 1) [40]. MRCP also allows evaluation of the main pancreatic duct (MPD) in patients with suspected AIP; diffuse irregular narrowing of the MPD or a long stricture $(>1 / 3$ the length of the MPD) with a lack of upstream dilatation are both suggestive of AIP [41]. IgG4-SC may present with no identifiable biliary stricture on cross-sectional imaging or ERC, in which case endoscopic ultrasonography may play a role in further evaluation [42].

\section{Endoscopic retrograde cholangiography}

Endoscopic retrograde cholangiopancreatography (ER$\mathrm{CP}$ ) is a useful initial evaluation of $\mathrm{IgG}_{4}$-SC because tissue samples are acquired; the procedure also plays a therapeutic role. Cytological examination of samples from biliary brushings are essential to exclude malignancy in the setting of a dominant stricture, but are not sufficient for diagnosis of $\mathrm{IgG}_{4}-\mathrm{SC}$ [43]. Ampullary biopsies are technically simple and the tissue may exhibit features diagnostic of IgG4-RD. In one study, ampullary biopsies stained positive for $\mathrm{IgG}_{4}$ in $18 / 27$ patients (67\%) with symptomatic AIP, compared to no sample from patients without the disease [44]. Endobiliary fluoroscopic biopsies may also reveal the characteristic features of IgG4-SC [38]. Recent advances in cholangioscopy have al- 
Table 2. Comparison of PSC and IgG4-SC

\begin{tabular}{lll}
\hline & \multicolumn{1}{c}{ PSC } & IgG4-SC \\
\hline Sex, male:female & $1.5: 1$ & $7: 1$ \\
Age of onset & Young $(<40)$ & Older $(>50)$ \\
Presentation & Cholestatic liver biochemistry & Obstructive jaundice \\
Biliary abnormalities & $\begin{array}{l}\text { Beading, short band-like strictures, } \\
\text { peripheral pruning }\end{array}$ & $\begin{array}{c}\text { Long smooth strictures, low CBD stricture, } \\
\text { prestenotic dilatation }\end{array}$ \\
Raised serum IgG4 levels & $<20 \%$ & $>70 \%$ \\
Pancreatic involvement & $<5 \%$ & $>80 \%$ \\
Multiorgan involvement & No & Yes \\
Association with IBD & $80 \%$ & $<10 \%$ \\
Histology & 'Onion-skin' fibrosis & Lymphoplasmacytic infiltrate, obliterative \\
& & phlebitis \\
\hline
\end{tabular}

Adapted from Joshi et al., with permission from John Wiley and Sons [50].

PSC, primary sclerosing cholangitis; IgG4-SC, immunoglobulin $\mathrm{G}_{4}$ sclerosing cholangitis; CBD, common bile duct; IBD, inflammatory bowel disease.

lowed the characteristic mucosal features of $\mathrm{IgG}_{4}$-SC to be evaluated via visually directed histological sampling [45]. A liver biopsy is sometimes diagnostically useful in patients with intrahepatic bile duct involvement or a hilar pseudotumor [36]. Biliary stenting may not be mandatory for all cases of obstructive jaundice secondary to known IgG4-SC and AIP. Corticosteroid therapy alone (thus without any need for biliary stenting) has been shown to be a safe and effective treatment for obstructive jaundice attributable to major biliary strictures in this group of patients $[46,47]$. Biliary stenting remains important for patients with unclear diagnoses or cholangitis.

\section{Differential diagnosis}

The cholangiographic features of $\mathrm{IgG}_{4}-\mathrm{SC}$ are similar to those of both PSC and CCA [6]; diagnosis based on ERC appearances alone may be unreliable [48]. The possibly confusing conditions are described separately below.

\section{Primary sclerosing cholangitis}

IgG4-SC accompanied by diffuse stenosis of the intrahepatic/proximal bile ducts (type 2 disease) is similar to PSC, being a progressive fibrosclerotic condition characterized by intra- and/or extra-hepatic structuring that does not respond to steroids. Expert interpretations of IgG4-SC and PSC cholangiograms suggest that the cholangiographic features revealed by ERCP alone are associated with a high IgG4-SC diagnostic specificity (88\%) but a low sensitivity (45\%) [49]. Cholangiographic features that may favor a diagnosis of $\mathrm{IgG}_{4}$-SC rather than PSC include longer strictures and the presence of both intra-hepatic and low bile duct strictures; the strictures of PSC tend to be shorter and the biliary tree appears beaded. Apart from bile duct stenosis, IgG4-SC may be associated with circular symmetrical thickening of the bile duct wall, smooth outer and inner wall margins, and homogenous internal echoing on abdominal CT, MRI, endoscopic ultrasound, and intraductal ultrasonography [36]. These features are apparent not only in stenotic areas but also in areas lacking stenosis (that thus appear normal on cholangiograms). Dynamic changes in biliary stricturing over weeks or months, steroid responsiveness, and the presence of associated pancreatic abnormalities, all favor a diagnosis of $\mathrm{IgG}_{4}$ SC compared to PSC. Other relevant factors are listed in Table 2 [50].

Elevated serum $\mathrm{IgG}_{4}$ levels have been recorded in up to $15 \%$ of patients with PSC; therefore, the serum IgG4 level does not appear to be helpful when seeking to differentiate PSC from IgG4-RD [20]. It remains unclear whether PSC and $\mathrm{IgG}_{4}$-SC represent different ends of the same disease spectrum or separate clinical entities. It may be reasonable to trial corticosteroids in all pa- 


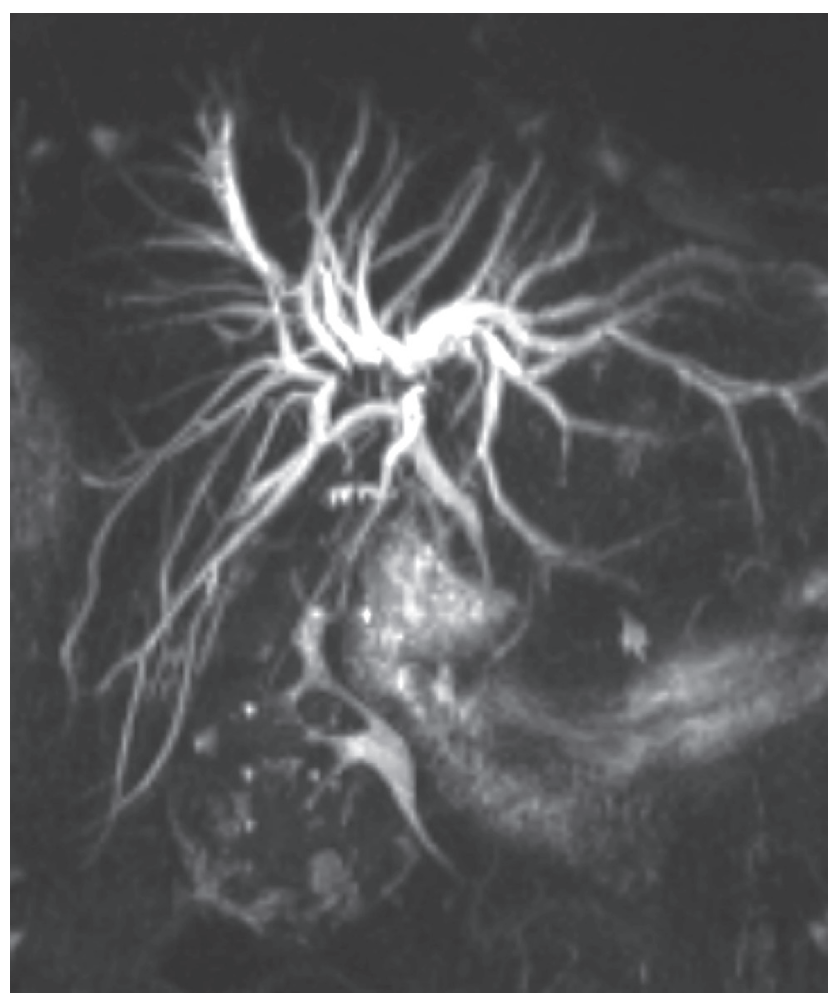

Figure 2. Cholangiogram showing central hilar stricturing secondary to immunoglobulin $\mathrm{G}_{4}$ sclerosing cholangitis disease.

tients with presumed PSC and elevated serum $\mathrm{IgG}_{4}$ levels, to assess the extent of cholangiopathic reversibility.

\section{Cholangiocarcinoma}

$\mathrm{IgG}_{4}-\mathrm{SC}$ with isolated stenosis of the distal common bile duct (type 1 disease) may be difficult to differentiate from distal CCA, whereas IgG4-SC accompanied by stenosis of the hilar hepatic bile duct (types 3 and 4 disease) can be difficult to differentiate from hilar CCA (Fig. 2) [36]. A review of 185 patients who underwent liver resection to treat presumed hilar CCA found that $17 \%$ had no evidence of malignancy, and of these, almost 50\% exhibited histological features consistent with those of $\mathrm{IgG}_{4}$-SC [51]. Only a few studies have explored how to differentiate $\mathrm{IgG}_{4}-\mathrm{SC}$ from $\mathrm{CCA}[52]$. IgG4-SC is particularly difficult to differentiate from hilar CCA when the former condition is not associated with AIP, or when the AIP diagnosis is unclear [53]. The vastly different treatment algorithms appropriate for IgG4-SC and CCA render accurate and timely differentiation essential.

In cases with suspect CCA, when ERCP fails to yield confirmatory cytological data, cholangioscopy plays a role in differentiating CCA from IgG4-SC. Peroral cholangioscopy accompanied by visually targeted biopsies affords a greater diagnostic accuracy than standard ERCP (with non-targeted biopsies) [54]. In a multicenter prospective study on 105 patients, the sensitivity and specificity of visual impressions of malignancy during cholangioscopy were $90 \%$ and $96 \%$, respectively. The sensitivity and specificity of cholangioscopy guided biopsies in terms of malignancy diagnoses were $85 \%$ and $100 \%$, respectively [55]. A later SRMA (10 studies, $\mathrm{n}=456$ ) reported pooled sensitivity and specificity of $66 \%$ and $98 \%$ for CCA diagnosis by cholangioscopic biopsy [56]. Thus, cholangioscopy has a particular role to play when ERCP alone fails to yield confirmatory cytological data, and the diagnosis thus remains unclear. Neither the serum $\mathrm{IgG}_{4}$ nor carbohydrate antigen (CA) 19-9 level accurately distinguish $\mathrm{IgG}_{4}-\mathrm{SC}$ from CCA [57,58].

\section{Secondary sclerosing cholangitis}

Secondary sclerosing cholangitis is a chronic disease that is phenotypically, clinically, and cholangiographically similar to PSC, and therefore IgG4-SC. The descriptor encompasses a broad spectrum of diseases including infection (e.g., AIDS cholangiopathy), congenital conditions (e.g., Caroli's disease), trauma, bilestones, infiltrative disease (e.g., histiocytosis X), and vascular conditions (e.g., post-liver transplantation hepatic arterial thrombosis). The exact cause can usually be elucidated by clinical history-taking.

\section{Treatment}

The goals of IgG4-SC treatment are to prevent irreversible fibrosis and biliary sepsis, both of which complicate untreated IgG4-SC [59]. Spontaneous remission is well-documented in AIP patients but spontaneous improvement of IgG4-SC disease of types 2 to 4 appears to be unusual [6o]. The mainstay of treatment is a tapering course of corticosteroids, as supported by the abovementioned, recent, prospective, randomized placebo-controlled trial of the utility thereof in AIP patients [26]. In terms of IgG4-RD disease, a typical regimen might include prednisolone 30 to $40 \mathrm{mg}$ daily for 4 weeks, with initial tapering over 2 weeks (depending on the response), followed by tapering of $5 \mathrm{mg}$ /day over 3 to 4 months, before maintaining a dose of $5 \mathrm{mg} /$ day for 
36 months. A response is usually evident within 4 to 6 weeks, evidenced by normalization of liver biochemistry and improvements in bile duct strictures. Patients with biliary obstructions should be closely monitored during corticosteroid therapy for signs of biliary sepsis. Serum $\mathrm{IgG}_{4}$ levels often fall during corticosteroid therapy and may then normalize. A recent retrospective study on 527 patients with IgG4-SC found that 90\% exhibited excellent responses to corticosteroid therapy; the alkaline phosphatase levels fell to $<50 \%$ of the pre-treatment values in $87 \%$ of cases and biliary strictures were alleviated in $90 \%$ [37]. Another study, which defined remission as complete resolution of strictures and/or normalization of liver function, reported remission in $67 \%$ of IgG4-SC cases [4] (vs. 99\% of AIP cases) [61]. A non-response to steroid therapy may be characteristic of a less inflammatory, fibrotic "burnt-out" IgG4-SC phase, or an alternative condition.

\section{Relapse}

Patients with IgG4-SC are at high risk of relapse. In an early University College Hospital, London cohort of patients with IgG4-SC and AIP, 57\% (13/23) relapsed [27]. Other studies have reported similar relapse rates [61]. Factors predictive of relapse include the presence of proximal strictures (IgG4-SC of types 2 to 4 ) and high serum IgG4 levels [4,22]. In patients who relapse, the treatment options include up-titration or re-introduction of corticosteroids with or without the addition of second-line immunosuppressants. Azathioprine is the drug most commonly prescribed, although it remains unclear whether the addition of an immunomodulator to a low-dose steroid affords any clinical benefit in terms of extending the time to further relapse. Another option is mycophenolate mofetil (750 to 1,000 mg twice daily). Factors predictive of resistance to immunomodulators include other organ involvement (thus, disease other than $\mathrm{IgG}_{4}-\mathrm{SC}$ ) and retroperitoneal fibrosis [30]. Rituximab effectively treated $\mathrm{IgG}_{4}$-SC in patients with aggressive disease; those who were intolerant of immunomodulators; and those who exhibited steroid-dependence. A nationwide French study of 33 patients (including those with IgG4-SC) recorded clinical responses in 29/31 symptomatic patients (93.5\%). Glucocorticoid withdrawal was achieved by 17 patients (51.5\%) [29]. Another prospective open-label study that included patients with IgG4-SC given rituximab found that $97 \%$ achieved a response, $77 \%$ were able to stop steroids and had not relapsed by 6 months later, and 46\% were in remission at 12 months [31]. As experience grows, a more top-down approach may be considered in high-risk patients (such as those with multi-organ or steroid-resistant disease); it may be best to target disease early to prevent fibrotic complications [5].

\section{CONCLUSIONS}

IgG4-RD is a rare and under-recognized multi-systemic disease; IgG4-SC is the biliary manifestation thereof. It is important to consider IgG4-RD in any patient presenting with obstructive jaundice or tumefactive lesions. IgG4-SC may mimic a number of other conditions including PSC and CCA; therefore, a high index of suspicion is required. Our understanding of the pathophysiological processes underlying IgG4-SC has improved dramatically in recent years, but the precise mechanism underlying this fibroinflammatory condition remains enigmatic. Recently, novel diagnostic and therapeutic strategies have markedly improved the management of patients with $\mathrm{IgG}_{4}$-SC. Ongoing research and future clinical studies are likely to further shape our understanding of this recently described disease.

\section{Conflict of interest}

No potential conflict of interest relevant to this article was reported.

\section{Acknowledgments}

This work was performed at UCLH/UCL that receives a portion of its funding from the Biomedical Research Center of the National Institute for Health Research (NIHR) of the Department of Health.

\section{REFERENCES}

1. Deshpande V, Zen Y, Chan JK, et al. Consensus statement on the pathology of IgG4-related disease. Mod Pathol 2012;25:1181-1192.

2. Brito-Zeron P, Ramos-Casals M, Bosch X, Stone JH. The clinical spectrum of IgG4-related disease. Autoimmun 
Rev 2014;13:1203-1210.

3. Kawa S. Current concepts and diagnosis of IgG4-related pancreatitis (type 1 AIP). Semin Liver Dis 2016;36:257-273.

4. Ghazale A, Chari ST, Zhang L, et al. Immunoglobulin G4-associated cholangitis: clinical profile and response to therapy. Gastroenterology 2008;134:706-715.

5. Culver EL, Chapman RW. IgG4-related hepatobiliary disease: an overview. Nat Rev Gastroenterol Hepatol 2016;13:601-612.

6. Okazaki K, Uchida K, Koyabu M, Miyoshi H, Takaoka M. Recent advances in the concept and diagnosis of autoimmune pancreatitis and IgG4-related disease. J Gastroenterol 2011;46:277-288.

7. Bozzalla Cassione E, Stone JH. IgG4-related disease. Curr Opin Rheumatol 2017;29:223-227.

8. Della Torre E, Bozzolo EP, Passerini G, Doglioni C, Sabbadini MG. IgG4-related pachymeningitis: evidence of intrathecal $\mathrm{IgG}_{4}$ on cerebrospinal fluid analysis. Ann Intern Med 2012;156:401-403.

9. Mattoo H, Mahajan VS, Della-Torre E, et al. De novo oligoclonal expansions of circulating plasmablasts in active and relapsing IgG4-related disease. J Allergy Clin Immunol 2014;134:679-687.

10. Trampert DC, Hubers LM, van de Graaf SFJ, Beuers U. On the role of $\mathrm{IgG}_{4}$ in inflammatory conditions: lessons for IgG4-related disease. Biochim Biophys Acta 2018;1864(4 Pt B):1401-1409.

11. Mattoo H, Mahajan VS, Maehara T, et al. Clonal expansion of $\mathrm{CD}_{4}(+)$ cytotoxic $\mathrm{T}$ lymphocytes in patients with IgG4-related disease. J Allergy Clin Immunol 2016;138:825838 .

12. Akiyama M, Suzuki K, Yamaoka K, et al. Number of circulating follicular helper $2 \mathrm{~T}$ cells correlates with $\mathrm{IgG}_{4}$ and interleukin-4 levels and plasmablast numbers in IgG4-related disease. Arthritis Rheumatol 2015;67:2476-2481.

13. Akiyama M, Yasuoka H, Yamaoka K, et al. Enhanced $\mathrm{IgG}_{4}$ production by follicular helper $2 \mathrm{~T}$ cells and the involvement of follicular helper $1 \mathrm{~T}$ cells in the pathogenesis of IgG4-related disease. Arthritis Res Ther 2016;18:167.

14. Gerli R, Schillaci G, Giordano A, et al. CD4+CD28- T lymphocytes contribute to early atherosclerotic damage in rheumatoid arthritis patients. Circulation 2004;109:27442748.

15. Wolfson AR, Hamilos DL. Recent advances in understanding and managing IgG4-related disease. F100oRes 2017;6(F1000 Faculty Rev):185.
16. Masamune A, Kikuta K, Nabeshima T, et al. Nationwide epidemiological survey of early chronic pancreatitis in Japan. J Gastroenterol 2017;52:992-1000.

17. Chari ST, Smyrk TC, Levy MJ, et al. Diagnosis of autoimmune pancreatitis: the Mayo Clinic experience. Clin Gastroenterol Hepatol 2006;4:1010-1016.

18. Huggett MT, Culver EL, Kumar M, et al. Type 1 autoimmune pancreatitis and IgG4-related sclerosing cholangitis is associated with extrapancreatic organ failure, malignancy, and mortality in a prospective UK cohort. Am J Gastroenterol 2014;109:1675-1683.

19. Oseini AM, Chaiteerakij R, Shire AM, et al. Utility of serum immunoglobulin $\mathrm{G}_{4}$ in distinguishing immunoglobulin $\mathrm{G}_{4}$-associated cholangitis from cholangiocarcinoma. Hepatology 2011;54:940-948.

20. Boonstra K, Culver EL, de Buy Wenniger LM, et al. Serum immunoglobulin $\mathrm{G}_{4}$ and immunoglobulin $\mathrm{G}_{1}$ for distinguishing immunoglobulin $\mathrm{G}_{4}$-associated cholangitis from primary sclerosing cholangitis. Hepatology 2014;59:1954-1963.

21. Mendes FD, Jorgensen R, Keach J, et al. Elevated serum $\mathrm{IgG}_{4}$ concentration in patients with primary sclerosing cholangitis. Am J Gastroenterol 2006;101:2070-2075.

22. Culver EL, Sadler R, Simpson D, et al. Elevated serum $\mathrm{IgG}_{4}$ levels in diagnosis, treatment response, organ involvement, and relapse in a prospective IgG4-related disease UK cohort. Am J Gastroenterol 2016;111:733-743.

23. Cheuk W, Chan JK. Lymphadenopathy of IgG4-related disease: an underdiagnosed and overdiagnosed entity. Semin Diagn Pathol 2012;29:226-234.

24. Strehl JD, Hartmann A, Agaimy A. Numerous IgG4-positive plasma cells are ubiquitous in diverse localised non-specific chronic inflammatory conditions and need to be distinguished from IgG4-related systemic disorders. J Clin Pathol 2011;64:237-243.

25. Zhang J, Chen H, Ma Y, et al. Characterizing IgG4-related disease with 18F-FDG PET/CT: a prospective cohort study. Eur J Nucl Med Mol Imaging 2014;41:1624-1634.

26. Masamune A, Nishimori I, Kikuta K, et al. Randomised controlled trial of long-term maintenance corticosteroid therapy in patients with autoimmune pancreatitis. Gut 2017;66:487-494.

27. Sandanayake NS, Church NI, Chapman MH, et al. Presentation and management of post-treatment relapse in autoimmune pancreatitis/immunoglobulin $\mathrm{G}_{4}$-associated cholangitis. Clin Gastroenterol Hepatol 2009;7:1089- 
1096.

28. Kamisawa T, Zen Y, Pillai S, Stone JH. IgG4-related disease. Lancet 2015;385:1460-1471.

29. Ebbo M, Grados A, Samson M, et al. Long-term efficacy and safety of rituximab in $\mathrm{IgG}_{4}$-related disease: data from a French nationwide study of thirty-three patients. PLoS One 2017;12:e0183844.

30. Hart PA, Topazian MD, Witzig TE, et al. Treatment of relapsing autoimmune pancreatitis with immunomodulators and rituximab: the Mayo Clinic experience. Gut 2013;62:1607-1615.

31. Carruthers MN, Topazian MD, Khosroshahi A, et al. Rituximab for IgG4-related disease: a prospective, open-label trial. Ann Rheum Dis 2015;74:1171-1177.

32. Brito-Zeron P, Kostov B, Bosch X, Acar-Denizli N, Ramos-Casals M, Stone JH. Therapeutic approach to IgG4-related disease: a systematic review. Medicine (Baltimore) 2016;95:e4002.

33. Sah RP, Chari ST, Pannala R, et al. Differences in clinical profile and relapse rate of type 1 versus type 2 autoimmune pancreatitis. Gastroenterology 2010;139:140-148.

34. Ahn SS, Song JJ, Park YB, Lee SW. Malignancies in Korean patients with immunoglobulin $\mathrm{G}_{4}$-related disease. Int J Rheum Dis 2017;20:1028-1035.

35. Okazaki K, Uchida K, Miyoshi H, Ikeura T, Takaoka M, Nishio A. Recent concepts of autoimmune pancreatitis and IgG4-related disease. Clin Rev Allergy Immunol 2011;41:126-138.

36. Ohara H, Okazaki K, Tsubouchi H, et al. Clinical diagnostic criteria of IgG4-related sclerosing cholangitis 2012. J Hepatobiliary Pancreat Sci 2012;19:536-542.

37. Tanaka A, Tazuma S, Okazaki K, et al. Clinical features, response to treatment, and outcomes of $\mathrm{IgG}_{4}$-related sclerosing cholangitis. Clin Gastroenterol Hepatol 2017;15:920-926.

38. Oh HC, Kim MH, Lee KT, et al. Clinical clues to suspicion of IgG4-associated sclerosing cholangitis disguised as primary sclerosing cholangitis or hilar cholangiocarcinoma. J Gastroenterol Hepatol 2010;25:1831-1837.

39. Yata M, Suzuki K, Furuhashi N, Kawakami K, Kawai Y, Naganawa S. Comparison of the multidetector-row computed tomography findings of IgG4-related sclerosing cholangitis and extrahepatic cholangiocarcinoma. Clin Radiol 2016;71:203-210.

40. Nakazawa T, Ohara H, Sano H, Ando T, Joh T. Schematic classification of sclerosing cholangitis with autoimmune pancreatitis by cholangiography. Pancreas 2006;32:229.

41. Kanno A, Masamune A, Shimosegawa T. Endoscopic approaches for the diagnosis of autoimmune pancreatitis. Dig Endosc 2015;27:250-258.

42. Shimizu S, Naitoh I, Nakazawa T, et al. IgG4-related sclerosing cholangitis with no biliary stricture but severe thickening of the bile duct wall. Intern Med 2016;55:15751579 .

43. Naitoh I, Nakazawa T, Kato A, et al. Predictive factors for positive diagnosis of malignant biliary strictures by transpapillary brush cytology and forceps biopsy. J Dig Dis 2016;17:44-51.

44. Kubota K, Kato S, Akiyama T, et al. Differentiating sclerosing cholangitis caused by autoimmune pancreatitis and primary sclerosing cholangitis according to endoscopic duodenal papillary features. Gastrointest Endosc 2008;68:1204-1208.

45. Okano N, Igarashi Y, Kishimoto Y, Ito K, Sasai D. Case of immunoglobulin $\mathrm{G}_{4}$-related cholangitis accompanying autoimmune pancreatitis: diagnosis by peroral cholangioscopy and treatment by endoscopic biliary stenting. Dig Endosc 2012;24 Suppl 1:62-66.

46. Bi Y, Hart PA, Law R, et al. Obstructive jaundice in autoimmune pancreatitis can be safely treated with corticosteroids alone without biliary stenting. Pancreatology 2016;16:391-396.

47. Arora Z, Chahal P. Comment re: "Obstructive jaundice in autoimmune pancreatitis can be safely treated with corticosteroids alone without biliary stenting". Pancreatology 2016;16:689-69o.

48. Kalaitzakis E, Webster GJ. Endoscopic diagnosis of biliary tract disease. Curr Opin Gastroenterol 2012;28:273-279.

49. Kalaitzakis E, Levy M, Kamisawa T, et al. Endoscopic retrograde cholangiography does not reliably distinguish IgG4-associated cholangitis from primary sclerosing cholangitis or cholangiocarcinoma. Clin Gastroenterol Hepatol 2011;9:800-803.

50. Joshi D, Webster GJ. Biliary and hepatic involvement in IgG4-related disease. Aliment Pharmacol Ther 2014;40:12511261.

51. Erdogan D, Kloek JJ, ten Kate FJ, et al. Immunoglobulin G4-related sclerosing cholangitis in patients resected for presumed malignant bile duct strictures. Br J Surg 2008;95:727-734.

52. Tabata T, Kamisawa T, Hara S, et al. Differentiating immunoglobulin g4-related sclerosing cholangitis from 
hilar cholangiocarcinoma. Gut Liver 2013;7:234-238.

53. Hamano H, Kawa S, Uehara T, et al. Immunoglobulin G4-related lymphoplasmacytic sclerosing cholangitis that mimics infiltrating hilar cholangiocarcinoma: part of a spectrum of autoimmune pancreatitis? Gastrointest Endosc 2005;62:152-157.

54. Ramchandani M, Reddy DN, Gupta R, et al. Role of single-operator peroral cholangioscopy in the diagnosis of indeterminate biliary lesions: a single-center, prospective study. Gastrointest Endosc 2011;74:511-519.

55. Navaneethan U, Hasan MK, Kommaraju K, et al. Digital, single-operator cholangiopancreatoscopy in the diagnosis and management of pancreatobiliary disorders: a multicenter clinical experience (with video). Gastrointest Endosc 2016;84:649-655.

56. Navaneethan U, Hasan MK, Lourdusamy V, Njei B, Varadarajulu S, Hawes RH. Single-operator cholangioscopy and targeted biopsies in the diagnosis of indeterminate biliary strictures: a systematic review. Gastrointest Endosc 2015;82:608-614.

57. Hirano K, Shiratori Y, Komatsu Y, et al. Involvement of the biliary system in autoimmune pancreatitis: a follow-up study. Clin Gastroenterol Hepatol 2003;1:453-464.

58. Straub BK, Esposito I, Gotthardt D, et al. IgG4-associated cholangitis with cholangiocarcinoma. Virchows Arch 2011;458:761-765.

59. Khosroshahi A, Wallace ZS, Crowe JL, et al. International consensus guidance statement on the management and treatment of IgG4-related disease. Arthritis Rheumatol 2015;67:1688-1699.

6o. Kamisawa T, Shimosegawa T, Okazaki K, et al. Standard steroid treatment for autoimmune pancreatitis. Gut 2009;58:1504-1507.

61. Hart PA, Kamisawa T, Brugge WR, et al. Long-term outcomes of autoimmune pancreatitis: a multicentre, international analysis. Gut 2013;62:1771-1776. 